\section{No clinical or biological difference between Chikungunya and Dengue Fever during the 2010 Gabonese outbreak}

\author{
Dieudonné Nkoghe, ${ }^{1,2}$ \\ Roland Fabrice Kassa Kassa, ${ }^{1}$ \\ Ulrich Bisvigou, ${ }^{3}$ Mélanie Caron, ${ }^{1}$ \\ Gilda Grard, ${ }^{1}$ Eric Maurice Leroy ${ }^{1,4}$ \\ 'Unité des Maladies Virales Emergentes, \\ Centre International de Recherches \\ Médicales de Franceville (CIRMF), \\ Franceville; ${ }^{2}$ Ministère de la Santé \\ Publique, Libreville; ${ }^{3}$ Unité de Biologie \\ Médicale et de Santé Publique, Centre \\ International de Recherches Médicales de \\ Franceville, Gabon; MIVEGEC, \\ Montpellier, France
}

\section{Abstract}

Chikungunya (CHIKV) and Dengue (DENV) viruses, both arboviruses, have caused multiple outbreaks worldwide. Their clinical features are poorly described in Africa and there is no comparative study, although Chikungunya is considered as a dengue-like disease. We conducted a comparative study of clinical and biological data from CHIKV and DENV positive patients during the 2010 Gabonese outbreak. Patients consulting with general symptoms and having laboratory confirmation for CHIKV or DENV were included. Clinical and biological data were recorded. Statistical analyses were performed using Epi Info. A P value $<0.05$ was considered significant. In all, $270 \mathrm{CHIKV+,} 53$ DENV+ and 20 co-infected patients were included in the study. Headaches, hemorrhage, leukopenia and lymphopenia were significantly (P respectively $0.01,0.001,0.02$ and 0.001 ) more frequent in DENV+ patients than in $\mathrm{CHIKV+.} \mathrm{There} \mathrm{was} \mathrm{no} \mathrm{additive} \mathrm{effect} \mathrm{of} \mathrm{the}$ two viruses.These clinical and hematological disorders are non specific and cannot assist for the differential diagnosis. These diseases are clinically indistinguishable, and need for laboratory confirmation.

\section{Introduction}

Chikungunya (CHIKV) and Dengue (DENV which is divided into 4 serotypes, 1 to 4 ) viruses are both arthropod-borne viruses belonging respectively to the genus Alphavirus, Togaviridae family and Flavivirus, Flaviviridae family, and transmitted to humans via a bite of infected Aedes mosquitoes (Aedes aegypti, Aedes albopictus)

Over the past two decades, they have caused multiple worldwide outbreaks, particularly in tropical and sub tropical areas. Recent CHIKV outbreaks started in Kenya in 2004, ${ }^{1,2}$ spread over the Indian Ocean region, ${ }^{3-7}$ reached India causing in all more than 1.5 million cases. ${ }^{8}$ Then, numerous imported cases were reported in different parts of the world, ${ }^{9}$ an outbreak occurred in Italy, ${ }^{10}$ while autochthonous cases were reported in France. ${ }^{11}$ In central Africa, outbreaks occurred in Democratic Republic of Congo, Cameroon, Gabon and Republic of Congo. ${ }^{12-15}$

As for Dengue fever (DF), its incidence has grown dramatically around the world, rising more than 30 -fold in recent decades. Two fifths of the world's population are at risk of infection, and WHO currently estimates there are 50 million dengue infections each year. The disease is now endemic in more than 100 countries in Africa, the Americas, the Eastern Mediterranean, South-east Asia and the Western Pacific. During the last decade, DENV epidemics have occurred in some East and West African countries, including Cape Verde where more than $17,000 \mathrm{DF}$ cases due to serotype 3 were notified. ${ }^{16}$

In practice, the global picture of both Chikungunya and DF is similar in their classical forms, so they can be confused particularly in areas where they cocirculate. ${ }^{17,18}$ After variable incubation period, both diseases start abruptly with acute fever, arthralgias, myalgias, headaches, rash and fatigue. Arthralgias are often incapacitating in CHIKV infection and can persist for several years in some patients. For DENV infection, after the initial febrile phase, hemorrhagic manifestations can occur, leading to Dengue Hemorrhagic Fever (DHF) indeed Dengue with Shock Syndrome (DSS). And finally, in addition to DENV, reports of deaths in La Reunion Island were directly attributed to $\mathrm{CHIKV}$, $^{71}$

Clinical features of CHIKV and DENV infections are poorly described in Sub Saharan Africa. Furthermore, there is no comparative study of the two infections, still lesser in a concurrent outbreak.

Here, we compare the clinical features and the laboratory findings for 343 laboratory-confirmed patients from the concurrent CHIKV/ DENV outbreak which occurred in Gabon in 2010.

\section{Materials and Methods}

\section{Study area and setting}

Franceville, the main town of the Haut Ogooue province, $512 \mathrm{~km}$ south-east from Libreville (capital of Gabon), is located at alti-
Correspondence: Dieudonné Nkoghe, Unité des Maladies Virales Emergentes, Centre International de Recherches Médicales de Franceville (CIRMF), BP 769 Franceville, Gabon. Tel. +241.0751.9100 - Fax: +241.677.295

E-mail: dnkoghe@hotmail.com

Key words: Chikungunya- Dengue- SymptomsGabon.

Acknowledgments: we thank Philippe Yaba André Delicat, Philippe Engandja, Yvette Lekibi, Loumbangoye Germaine, Koné Martine and Mouyabi Victoire from Centre International de Recherches Médicales de Franceville (CIRMF), Gabon, for their technical assistance. CIRMF is supported by the Gabonese State, Total Gabon, and Ministère Français de la Coopération.

Contributions: EML and DN conceived and designed the experiments; DN, RFK, MC, GG and UB performed the experiments, UB contributed reagents, materials and analytical tools; DN analyzed the data; DN wrote the paper.

Conflict of interest: the authors report no conflicts of interest.

Received for publication: 14 September 2011. Revision received: 29 0ctober 2011.

Accepted for publication: 27 December 2011.

This work is licensed under a Creative Commons Attribution NonCommercial 3.0 License (CC BYNC 3.0).

CC Copyright D. Nkoghe et al., 2012

Licensee PAGEPress, Italy

Infectious Disease Reports 2012; 4:e5

doi:10.4081/idr.2012.e5

tude 333 meters, at the end of the TransGabon railway and the national 3 road, in a savannah ecosystem constituted by forest gallery (Figure 1). There are 5 health care capacities (HCC) including 2 public hospitals with 170 beds in all and the medical unit of the Centre International de Recherches Medicales de Franceville (CIRMF). A concomitant CHIKV/ DENV outbreak raged 11 towns and one village of the southeast, from February to July 2010, and recorded 1127 laboratory confirmed cases in Franceville. The CIRMF was included as a partner of the $\mathrm{MoH}$, in the response to this outbreak, and implicated in active surveillance, case management and diagnostic. As part of these activities, a prospective study was initiated, and the investigations were considered as a component of the public health response and authorized by the Regional Health Director.

\section{Study population and data collection}

At the time of the outbreak, the MoH provided a case definition including 2 categories: a 
suspected case was a patient presenting with at least one of the following symptoms: fever, arthralgias, myalgias, headaches, rash, fatigue, nausea, vomiting, diarrhea, bleeding, and jaundice; a confirmed case was a suspected case with laboratory confirmation.

All suspected cases consulting in the CIRMF medical unit were examined and demographic and clinical data collected in standardized questionnaire. An oral consent was required for the blood sampling.

\section{Laboratory analyses}

Blood samples were collected in two 7-mL EDTA Vacutainer tubes and one 7-mL dry tube (VWR International, France). The tubes were stored in the dark at $+4^{\circ} \mathrm{C}$ until arrival at the laboratory. Plasma was obtained by centrifugation (10 min, $2000 \mathrm{~g}$ ).

Thick and thin blood films were stained with $20 \%$ Giemsa and examined for malaria parasites. Patients with positive test were excluded from the study. Hematological (Hematology Analyser ACT 10, Beckman Coulter) and biochemical (creatinine, AST, ALT) tests were performed (Automatic Analyser Hitachi model 902, Roche Diagnostics). Samples were tested for CHIKV and DENV RNA genomes by using TaqMan qRT-PCR technology and specific primers and probes. ${ }^{14}$ When used during the acute phase (1-7 days after onset of symptoms), the TaqMan qRT-PCR technology has a sensitivity of up to $80 \%$ and specificity of $100 \% .{ }^{19,20}$ Co-infected CHIKV+/DENV+ patients were included in this study.

\section{Statistical analyses}

Statistical analyses were performed using Epi Info software (6.04, Epiconcept). Results were expressed as averages (with their SD) and percentages (with their 95\% confidence interval, IC). The Student's $t$ test was used to compare laboratory parameters and continuous clinical variables. For qualitative variables, the Chi square test or Fisher's exact test were used as appropriate. A P value $<0.05$ was considered to denote statistical significance.

\section{Results}

Totally, 433 suspected individuals consulted at CIRMF during the study period. They represented $30 \%$ of the laboratory confirmed cases of Franceville. The sex ratio M/F was 0.99 and the mean age was $30 \pm 15$ (range, 1-77 years). Among these 433 patients, 343 (79.2\%) were laboratory confirmed with $270 \quad(62.3 \%)$ CHIKV+, 53 (12.2\%) DENV+. There were 20 CHIV/DENV+ co-infected patients. CHIKV+ and DENV+ patients represented respectively $78.7 \%$ and $15.4 \%$ of the total positive samples.
The sex ratio $\mathrm{M} / \mathrm{F}$ was 0.85 for the $\mathrm{CHIKV}$ group, 0.55 for the DENV group and 1 for the CHIKV/DENV group, and the mean age was respectively $30 \pm 16$ (range $1-77$ years), $31 \pm 14$ (range, 5-76 years), and 36 \pm 12 (range, 1-74).

Hospitalization was required for 39 (14.4\%) CHIKV+, 2 (3.7\%) DENV+ ( $\mathrm{P}=0.02)$, and 6 CHIKV/DENV+ patients. There was no difference in the mean duration of symptoms $(\mathrm{P}=0.13)$.

Patients consulted on average 2 days after the onset of symptoms. The frequency of each symptom and biological parameter of the three groups was compared. Headaches and hemorrhage were more frequent ( $\mathrm{P}$ respectively 0.01 and 0.001 ) in the DENV+ group than in the CHIKV+ group (Table 1), and myalgias were more frequent in the CHIKV and DENV groups than in the CHIKV/DENV group $(\mathrm{P}=0.005$ and 0.02 respectively). Similarly, leukopenia and lymphopenia were more frequent ( $\mathrm{P}$ respectively 0.02 and 0.001 ) in the DENV+ group than in the CHIKV+ group (Table 2). There was no difference in biological parameters between the co-infected group and the other groups.

\section{Discussion}

We conducted a comparative study of clinical and biological data of patients with Chikungunya and Dengue viruses infections during a concurrent outbreak which occurred in south east Gabon in 2010. Of the 343 individuals included in the study, 270 were $\mathrm{CHIK}+$, 53 were $\mathrm{DENV}+$, and 20 were co-infected (CHIK+/DEN+). Headaches, hemorrhage, leukopenia and lymphopenia were significantly more frequent in DENV+ patients.

In our opinion, negative patients may be considered as having another disease. Thus, the circulation during this epidemic of other arboviruses such as West Nile, Rift Valley

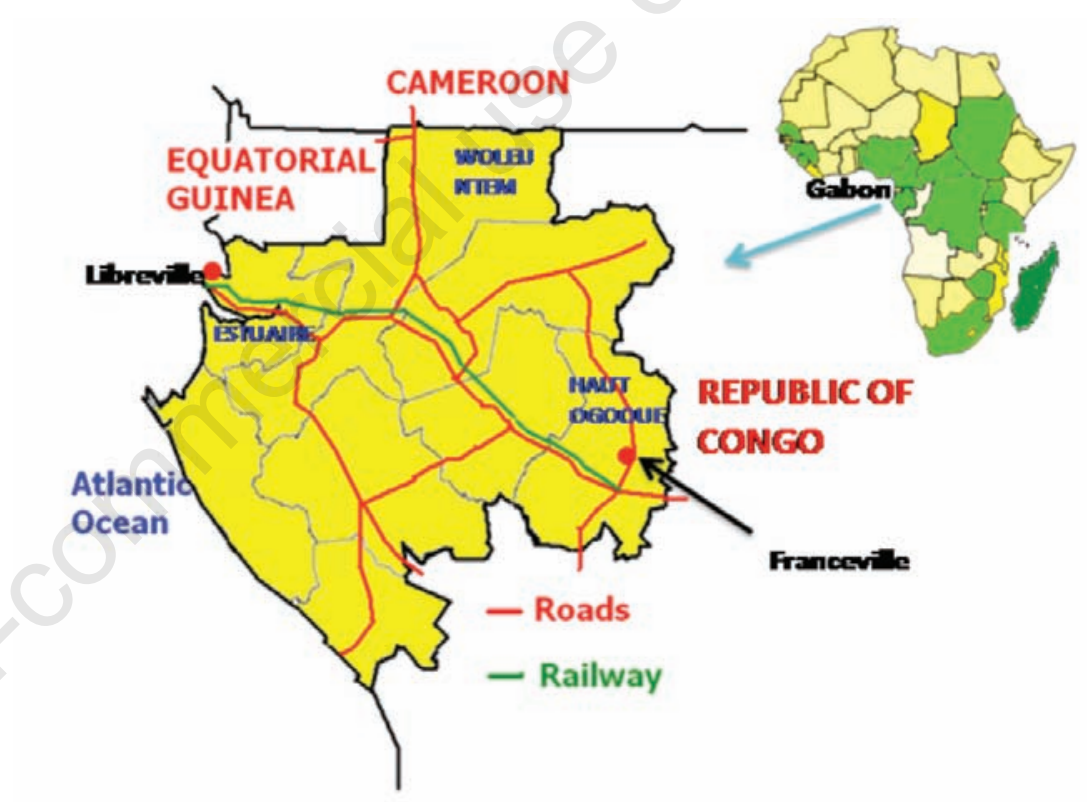

Figure 1. Map of Gabon with location of the Gabonese Chikungunya outbreak in 2010.

Table 1. Comparison of clinical signs between Chikungunya and Dengue-infected patients during the 2010 outbreak in Gabon.

\begin{tabular}{|c|c|c|c|c|c|c|}
\hline & \multicolumn{2}{|c|}{ CHIKV+ } & \multicolumn{2}{|c|}{ DENV+ } & \multirow[t]{2}{*}{$\mathbf{P}$} \\
\hline & & $\mathbf{N}$ & $\%$ & $\mathbf{N}$ & $\%$ & \\
\hline Hospitalization & 39 & 14.3 & 2 & 3.6 & 0.02 & \\
\hline \multirow[t]{9}{*}{ Clinical symptoms } & Fever & 234 & 86 & 52 & 92.9 & 0.16 \\
\hline & Arthralgia & 227 & 83.5 & 47 & 83.9 & 0.93 \\
\hline & Myalgia & 195 & 71.7 & 40 & 71.4 & 0.96 \\
\hline & Headache & 190 & 69.9 & 48 & 85.7 & 0.01 \\
\hline & Asthenia & 214 & 78.7 & 44 & 78.6 & 0.72 \\
\hline & Skin rash & 111 & 40.8 & 15 & 26.8 & 0.06 \\
\hline & Pruritus & 73 & 26.8 & 11 & 19.6 & 0.26 \\
\hline & Digestive signs & 87 & 32 & 19 & 34.5 & 0.86 \\
\hline & Hemorragy & 5 & 1.8 & 7 & 12.5 & 0.001 \\
\hline
\end{tabular}

CHIKV, Chikungunya virus; DENV, Dengue virus. 
Table 2. Comparison of biological parameters between Chikungunya and Dengue-infected patients during the 2010 outbreak in Gabon.

\begin{tabular}{llcccccc} 
& & \multicolumn{2}{c}{ CHIKV+ } & \multicolumn{2}{c}{ DENV+ } & P \\
Bean & SD & Mean & SD & \\
Biological parameters & Haemoglobin & 12.3 & 1.7 & 11.9 & 1.14 & 0.49 \\
& Leucocytes & 5243 & 1676 & 3390 & 1517 & 0.02 \\
\hline & Lymphocytes & 2228 & 216 & 1401 & 243 & 0.001 \\
& Platelets & 233089 & 81750 & 225600 & 60824 & 0.77 \\
\hline & ASAT & 17 & 11 & 18 & 10 & 0.38 \\
& ALAT & 45 & 35 & 50 & 34 & 0.58 \\
\hline & Créatinine & 96 & 32 & 88 & 32 & 0.14 \\
\hline
\end{tabular}

CHIKV, Chikungunya virus; DENV, Dengue virus.

Fever and Yellow Fever viruses, cannot be ruled out.

To our knowledge, this is the first comparative study of these two diseases. Previous descriptive studies focused on one or the other one. In these descriptions, both diseases are characterized by fever, headaches, arthralgias, myalgias and rash. ${ }^{3,5,7,13}$ All these symptoms are non specific. In addition, Chikungunya is described in the literature as a dengue-like disease, due to their common symptoms. Nevertheless, the existence of incapaciting joints pain located at the extremities seems specific of Chikungunya. This distinction is difficult in tropical areas where many other diseases, such as malaria, typhoid fever, influenza, share the same symptoms. ${ }^{17}$ Furthermore, in epidemic setting, their cases definitions overlap. So, in our field experience, these diseases are clinically indistinguishable, and the problem of differential diagnosis may be compounded during concomitant CHIKV/DENV outbreaks in a malaria endemic area. In their classical form, the hemorrhage constitutes an inconstant sign. Bleeding is generally minimal, non life-threathning, and cannot assist for the differential diagnosis. Its presence classifies Chikungunya and Dengue as hemorrhagic fever. To date, Dengue Hemorrhagic Fever has yet been described in Gabon. This entity is more recognizable, leading, with the shock syndrome, to the severity of the disease.

Our study showed that leukopenia and lymphopenia were significantly associated with DENV than CHIKV. Lymphocytopenia is probably due to apoptosis of peripheral lymphocytes. These hematological disorders are non specific and cannot also assist for the differential diagnosis.

Patients with mixed infection did not seem to have more severe symptoms or biological disorders, suggesting no additive effect of the two viruses.

Finally, there is no marked difference. The clinical and biological differences detected in our analysis are not useful in the field. So, our study confirms that the two diseases are clinically similar and need for laboratory confirmation for their recognition.

Since their appearance in 2007 in the north of the country, ${ }^{14}$ these viruses have spread in the south east, together with the vector Aedes albopitus. Gradual invasion of the entire country is foreseeable. Actually, only CIRMF can confirm the diagnosis. So, specific diagnosis tests are needed in all regional hospitals.

\section{References}

1. Kariuki Njenga M, Nderitu L, Ledermann JP, et al. Tracking epidemic Chikungunya virus into the Indian Ocean from East Africa. J Gen Virol 2008;89:2754-60.

2. Sergon K, Njuguna C, Kalani R, et al. Seroprevalence of Chikungunya virus (CHIKV) infection on Lamu Island, Kenya, October 2004. Am J Trop Med Hyg 2008;78: 333-7.

3. Sissoko D, Malvy D, Giry C, et al. Outbreak of Chikungunya fever in Mayotte, Comoros archipelago, 2005-2006. Trans R Soc Trop Med Hyg 2008;102:780-6.

4. Beesoon S, Funkhouser E, Kotea N, et al. Chikungunya fever, Mauritius, 2006. Emerg Infect Dis 2008;14:337-8.

5. Ratsitorahina M, Harisoa J, Ratovonjato J, et al. Outbreak of dengue and Chikungunya fevers, Toamasina, Madagascar, 2006. Emerg Infect Dis 2008;14:1135-7. dengue, south-west Indian Ocean. Wkly Epidemiol Rec 2006;81:106-8.

7. Renault P, Solet JL, Sissoko D, et al. A
6. WHO. Outbreak news. Chikungunya and major epidemic of chikungunya virus infection on Reunion Island, France, 20052006. Am J Trop Med Hyg 2007;77:727-31.

8. WHO. Outbreak and spread of Chikungunya. Wkly Epidemiol Rec 2007,82:40915.

9. Powers AM, Logue $\mathrm{CH}$. Changing patterns of chikungunya virus: re-emergence of a zoonotic arbovirus. J Gen Virol 2007;88: 2363-77.

10. Rezza G, Nicoletti L, Angelini R, et al. Infection with chikungunya virus in Italy: an outbreak in a temperate region. Lancet 2007;370:1840-6.

11. Gould EA, Gallian P, De Lamballerie X, Charrel RN. First cases of autochthonous dengue fever and chikungunya fever in France: from bad dream to reality! Clin Microbiol Infect 2010;16:1702-4.

12. Muyembe-Tamfum JJ, Peyrefitte CN, Yogolelo R, et al. Epidemic of Chikungunya virus in 1999 and 200 in the Democratic Republic of the Congo. Med Trop 2003;63: 637-8.

13. Peyrefitte CN, Rousset D, Pastorino BA, et al. Chikungunya virus, Cameroon, 2006. Emerg Infect Dis 2007;13:768-71.

14. Leroy EM, Nkoghe D, Ollomo B, et al. Concurrent chikungunya and dengue virus infections during simultaneous outbreaks, Gabon, 2007. Emerg Infect Dis 2009;15:591-3.

15. Kelvin AA. Outbreak of Chikungunya in the Republic of Congo and the global picture. J Infect Dev Ctries 2011;5:441-4.

16. Franco L, Di Caro A, Carletti F, et al. Recent expansion of dengue virus serotype 3 in West Africa. Euro Surveill 2010;15:pii9460.

17. Pialoux G, Gaüzère BA, Jauréguiberry $S$, Strobel M. Chikungunya, an epidemic arbovirosis. Lancet Infect Dis 2007;7:31927.

18. Staples JE, Breiman RF, Powers AM. Chikungunya fever: an epidemiological review of a re-emerging infectious disease. Clin Infect Dis 2009;49:942-8.

19. Barkham TM, Chung YK, Tang KF, Ooi EE. The performance of RT-PCR compared with a rapid serological assay for acute dengue fever in a diagnostic laboratory. Trans R Soc Trop Med Hyg 2006;100:142-8.

20. Parida MM, Santhosh SR, Dash PK, et al. Rapid and real-time detection of Chikungunya virus by reverse transcription loop-mediated isothermal amplification assay. J Clin Microbiol 2007;45:351-7. 